\title{
Fetal Growth Restriction and Cardiovascular Health Among Adolescents
}

\author{
ANURAdha V Khadilkar AND LS Parthasarathy \\ From Hirabai Cowasji Jehangir Medical Research Institute, Jehangir Hospital, Pune, India. vamankhadilkar@gmail.com
}

I $n$ developing countries, low birth weight (LBW) is a major public health problem. Seventy-two percent of low birth weight infants in developing countries are born in Asia, with 40 per cent being born in India [1]. Fetal growth restriction or intrauterine growth restriction (IUGR) is a condition where the fetus fails to achieve its intrinsic growth potential, due to anatomical or functional disorders and diseases in the feto-placentalmaternal unit. More than $50 \%$ of LBW infants born in India are IUGR; thus, the burden of IUGR is high in India [2]. IUGR is associated not only with significant perinatal morbidity and mortality, but also with long-term complications throughout life [3]. Thus, addressing IUGR and related problems is critical in developing countries.

Being born IUGR is associated with increased risk of degenerative diseases in adulthood, including diabetes, hypertension, and cardiovascular diseases [4]. The association between IUGR and cardiovascular diseases was first described by 'developmental origins of adult disease' hypothesis, often called The Barker hypothesis, which proposes that these diseases originate through adaptations of the fetus when it is undernourished. These adaptations may be cardiovascular, metabolic, or endocrine, and they may permanently program the structure and function of the body, increasing coronary heart disease risk factors such as hypertension, type 2 diabetes mellitus, insulin resistance and hyperlipidemia.

Although the association between IUGR and adult disease has been extensively studied, reports also suggest that rather than merely IUGR, postnatal weight gain is in itself a risk factor for diseases in later life. Cole, using a life-course plot, has emphasized the substantial impact of later weight on high blood pressure in adolescence [5]. Further, Barker, et al. [6] also reported that the risk of coronary events was more strongly related to the tempo of childhood gain in body mass index (BMI) than to the BMI attained at any particular age. They also report that small size at birth, low BMI at 2 years of age, and high BMI at 11 years of age were each associated with later coronary events in both men and women [6]. Thus, it seems that apart from being IUGR, the change in trajectory of growth in infants born IUGR contributes to adult-onset diseases. These observations demonstrate that coronary events are independently associated with not only prenatal but postnatal growth as well. In line with these observations, a study conducted in India reported that thinness at 2 years of age, followed by a rapid increase in BMI, is associated with the development of impaired glucose tolerance and type 2 diabetes at approximately 30 years of age [7].

In the current issue of Indian Pediatrics, Alves, et al. [8] from Brazil reiterate the importance of studying fetal growth restricted babies and the associated complications. Though the authors could not demonstrate an association between fetal growth restriction and changes in cardiovascular risk factors in adolescents at this stage, they bring to our notice that the growth restricted group was similar to the non growth restricted group in the parameters studied. Their results possibly indicate that these adolescents had changed trajectory to equal the control group. Though the current paper is interesting, we think that the results would have been on more firm grounds if, given the age range and gender, comparisons between groups in anthropometric parameters would have been made after calculating $\mathrm{Z}$ scores. Assessing the change in trajectory, or lack of it, would have strengthened the authors' observations. As the authors have also discussed, one of the limitations of their study is that the tempo of the anthropometric characteristics is of greater importance than a single reading in adolescent years, and further follow-up of these children may possibly replicate the results, as previously reported. Additional longitudinal cohort studies are required to elucidate long-term impact of IUGR on cardiovascular disease in developing countries.

Funding: None; Competing interests: None stated.

\section{REFERENCES}

1. WHO. UNICEF. Low Birthweight: Country, Regional and Global Estimates. World Health Organization, Geneva, 2004.

2. Pinheiro A, David A, Joseph B. Pregnancy weight gain 


\section{EDITORIALS}

and its correlation to birth weight. Indian J Med Sci 2001; 55:266-70.

3. Cosmi E, Fanelli T, Visentin S, Trevisanuto D, Zanardo V. Consequences in infants that were intrauterine growth restricted. J Pregnancy. 2011;2011:364381. doi: 10.1155/ 2011/364381.

4. Alexe DM, Syridou G, Petridou ET. Determinants of early life leptin levels and later life degenerative outcomes. Clin Med Res. 2006;4:326-35.

5. Cole TJ. Modeling postnatal exposures and their interactions with birth size. J Nutr. 2004;134:201-4.

6. Barker DJ, Osmond C, Forsén TJ, Kajantie E, Eriksson
JG. Trajectories of growth among children who have coronary events as adults. N Engl J Med. 2005;353:18029.

7. Bhargava SK, Sachdev HS, Fall CHD, Osmond C, Lakshmy R, Barker DJ, et al. Relation of serial changes in childhood body-mass index to impaired glucose tolerance in young adulthood. N Engl J Med 2004;350:865-75.

8. Alves PJS, Henriques ACPT, Silva KF, Leite AJM, Feitosa FEL, Alencar CHM, et al. The influence of fetal growth restriction on cardiovascular health among adolescents in Brazil: A retrospective cohort study. Indian Pediatr. 2015;52:109-14. 